\title{
Use of telephone and web interfaces of interactive response technology at clinical investigator sites in clinical trials
}

\section{Saarela, Anna-Leena}

2019-06

Saarela , A-L , Walzer , A \& Juppo , A M 2019 , ' Use of telephone and web interfaces of interactive response technology at clinical investigator sites in clinical trials ' , Clinical trials : journal of the Society for Clinical Trials , vol. 16 , no. 3 , pp. 297-305 . https://doi.org/10.1177/1740774519832329

http://hdl.handle.net/10138/315657

https://doi.org/10.1177/1740774519832329

unspecified

acceptedVersion

Downloaded from Helda, University of Helsinki institutional repository.

This is an electronic reprint of the original article.

This reprint may differ from the original in pagination and typographic detail.

Please cite the original version. 
Use of telephone and web interfaces of Interactive Response Technology at clinical investigator sites in clinical trials

Short title: Use of IRT interfaces in clinical trials

Total number of words: 3974

Authors: Anna-Leena Saarela ${ }^{1,2}$, Anja Walzer ${ }^{3}$, Anne Mari Juppo ${ }^{1}$

${ }^{1}$ Division of Pharmaceutical Chemistry and Technology, Faculty of Pharmacy, Formulation and Industrial Pharmacy Unit, University of Helsinki, Helsinki, Finland

${ }^{2}$ Crown CRO Oy, Espoo, Finland

${ }^{3}$ Bayer AG, Development, Pharmaceuticals, Berlin, Germany

\section{Corresponding author:}

Anna-Leena Saarela, Crown CRO Oy, Vaisalantie 4, FI-02130 Espoo, Finland, tel.: +358-40-7676-968. Email: anna-leena.saarela@iki.fi

Abbreviations: IRT, Interactive Response Technology

IVR(S), Interactive Voice Response (System)

IWR(S), Interactive Web Response (System) 


\section{ABSTRACT}

Background: Interactive Response Technologies (IRT) are used in clinical trials to provide services such as automated randomization and medication logistics management. The objective of this paper is to investigate the usage of telephone (Interactive Voice Response, IVR) and web (Interactive Web Response, IWR) interfaces of IRT at clinical investigator sites in clinical trials, to obtain information about the preferences of IRT end users between the telephone and web interfaces, and to explore the relevance of the telephone interface in this setting.

Methods: The data consists of an online survey conducted in spring 2016 with clinical investigators, study nurses and pharmacists in 13 countries.

Results: Ninety-eight percent of survey respondents preferred the web interface over the telephone interface, the most important reason being superior usability. However, the respondents indicated the usability of IRT interfaces is not optimal, and lack of integration and consistency across systems is common. A vast majority of IRT end users at clinical sites prefer to use the web interface over the telephone interface, but most also feel there would need to be a back-up system.

Conclusions: Based on the results, it would be beneficial to improve the usability of the IRT interfaces, and to increase consistency across systems from the current level. Support to and training of the users, as well as clarifying the responsibilities between sites and the sponsor should also be a focal point. Study sponsors should explore with IRT service providers how removing the telephone interface would impact future studies, and whether there could be a more efficient means to achieve a reliable back-up to the web interface instead of a dedicated telephone interface. 
Keywords: Clinical trials, Interactive Response Technology, Interactive Voice Response, Interactive Web Response, User interface, Randomization 


\section{BACKGROUND}

Pharmaceutical companies utilize technological solutions to maintain data in clinical studies. ${ }^{1}$ Interactive Response Technologies (IRT) are used to provide services such as automated randomization, medication logistics management and drug supply optimization. As clinical IRT end users, physicians, study nurses, and pharmacists enter data for pre-defined data points into the IRT system, and the system gives an output, which could be a subject or randomization number allocated to the subject, or the number of a medication pack that is to be dispensed to the subject. In practice, the means of entering the data into the IRT system is either a telephone (Interactive Voice Response, IVR) or a web page on the Internet (Interactive Web Response, IWR). ${ }^{1}$ Currently, many studies utilize an IRT system consisting of both a telephone and a web interface, and the end user can choose freely which interface to use. The expectation is that the web interface is becoming more and more popular over the telephone interface that may be considered cumbersome by the end user compared with a web interface.

The use of IRT at the clinical investigator sites, the user experience, and the relevance of the telephone interface in the current setting has not been systematically studied until now. The aim of this study was to investigate the preferences of IRT end users between telephone and web interfaces, to gain a deeper understanding behind the reasons why one interface is preferred over the other. 


\section{METHODS}

A survey was conducted to investigate the attitudes and preferences of IRT end users at clinical sites. Earlier research has been limited to analysing qualitative data only, or investigating the adoption of various clinical trial technologies without addressing the preference of end users., ${ }^{2,3}$

The survey questionnaire was developed by the authors of this manuscript. The authors have several years of experience in working with IRT systems and clinical end users of IRT in clinical trials. The questionnaire was piloted in one country on two clinical investigators and one study nurse with extensive experience on IRT use.

The questionnaire consisted of 15 questions: closed-ended multiple-choice questions for quantitative data and open-ended questions for qualitative data concerning the reasons behind favouring one interface over the other. Demographic background data contained questions about the respondent's role in clinical studies, experience of using IRT systems, gender, age, work experience in clinical studies, and geographical location. Questions pertaining to IRT interfaces included items on whether the respondent would prefer to use the telephone or web interface if they could choose freely, including their perceived reasons for the choice. Those who responded that they would prefer to use the web interface were also asked whether they would wish to keep the telephone interface as a back-up option. The rationale for not asking telephone-preferring respondents

whether they would want to have a web interface as a back-up option is that the industry is gradually shifting more towards using the web, and therefore it would not be realistic 
to assume that the web interface would be dropped and replaced with telephone only in any current study. ${ }^{3}$

The target population of the survey was IRT users at clinical investigator sites: principal investigators, sub-investigators, study nurses, coordinators and study pharmacists. The contact details of the respondents were obtained from lists of registered IRT users of three clinical studies sponsored by Bayer and conducted in 2012-2015. The recipients were chosen from studies ongoing at the time and utilizing IRT systems provided by different service providers to gain a broader range of data to improve the generalizability of the results. Respondent countries were chosen to represent variability in geographical location within the restrictions of the Bayer global footprint, and to represent areas with different expected usage of the telephone interface.

The questionnaire was distributed to 1,388 recipients in 13 countries via electronic mail with a link to the online questionnaire. Contact details were invalid for 141 recipients, resulting in 1,247 delivered invitations to participate in the survey. The participating countries and their respective numbers of delivered invitations were: Austria (59), Belgium (89), Canada (52), Czech Republic (62), Denmark (55), Finland (46), France (98), Germany (189), Italy (49), Poland (61), Spain (81), United Kingdom (77), and USA (329). The first wave of invitations was sent on $22^{\text {rd }}$ March 2016, with two reminders in April 2016.

Quantitative and qualitative analyses were performed using the software packages IBM SPSS Statistics version 23 (IBM Corp, Armonk, NY, USA) and ATLAS.ti version 7 
(ATLAS.ti GmbH, Berlin, Germany), respectively. SPSS was used to analyse the data by descriptive statistics, frequency distributions and cross-tabulation. In crosstabulations, dependencies between the parameters were tested using the Chi-square test $\left(\mathrm{X}^{2}\right)$ or Fisher's exact test. ${ }^{4}$ If the p-value was less than 0.05 , the dependency between the parameters was considered statistically significant. Survey data was analysed qualitatively by thematic and grouping methods, using deductive and inductive analysis methods.

\section{RESULTS AND DISCUSSION}

A total of 231 responses were received with 199 completed responses and a total response rate of $19 \%$. Based on the demographic data (Table 1), 51\% of respondents were study nurses or coordinators, $22 \%$ study pharmacists and $21 \%$ principal investigators or sub-investigators. Eighty percent were female, as expected, as the study nurses often are the staff members mostly using the technological solutions at clinical sites, and females are also over-represented in the nursing role in many countries. ${ }^{5}$ Respondents who had not used an IRT system within the past five years were excluded $(n=11)$, as their experience was not considered recent enough for the purposes of this study.

The single most commonly used IRT system among the respondents was Parexel MyTrials / Perceptive ClinPhone, which $44 \%$ of the respondents were using at the time of the survey. The second most common systems used were ICON ICOPhone (20\%) and Almac IXRS (18\%). Six other proprietary IRT systems also received single 
mentions as the most common system being used by individual respondents. The IRT systems respondents reported using most often at the time of the survey are presented in Figure 1. The respondents also mentioned having experience of systems from 15 other IRT service providers, highlighting the fact that the commercial IRT field is highly fragmented.

When asked which IRT interfaces, i.e. the web, telephone or other interface, the respondents had used at least once, $98 \%(\mathrm{n}=211)$ reported having used the web and $47 \%(\mathrm{n}=101)$ the telephone interface at least once. In other words, more than half of the respondents reported they had never tried the telephone interface before. Also noteworthy is that, apart from the telephone and web, none of the respondents reported having used any other IRT interfaces, e.g. IRT mobile applications. This is most likely due to lack of opportunities, as IRT service providers are just starting to offer other interfaces in addition to telephone and web.

The most preferred IRT interface was the web, which was preferred by $98 \%$ of the respondents, while $2 \%$ would have preferred the telephone if they could choose freely. Of those preferring the telephone over the web, $80 \%$ were mostly using ICON's IRT system at the time of the survey $\left(\mathrm{X}^{2}[4]=12.8, \mathrm{p}=0.012\right.$, Pearson's Chi-square test $)$. Statistical tests showed no dependency between the preferred interface and respondent's age, gender, role or length of clinical experience.

The operational reliability of an IRT system can be considered crucial, as it is ethically necessary to provide correct and timely treatment to study subjects, and subject safety 
may require immediate access to the system. ${ }^{6}$ IRT systems are becoming more and more web-based, but there are situations where, for example, subject randomization may need to occur in an acute environment where no computers are accessible, or emergency unblinding may need to be carried out by non-study personnel. Therefore, those respondents who preferred the web over the telephone were asked whether they would wish to keep the telephone as a back-up interface in addition to the web. Of the respondents, $77 \%$ opted yes for keeping the telephone as back-up, and $23 \%$ felt it would not be necessary to keep the telephone as a back-up interface. This result can be considered surprising, but there was also geographical variation.

The desire to keep the telephone interface as back-up depended heavily on the respondent country $\left(\mathrm{X}^{2}[12]=41.1, \mathrm{p}<0.001\right.$, Pearson's Chi-square test $)$. In Italy, all respondents $(n=6)$ wanted to keep the telephone as back-up, while in Austria none of the respondents $(n=4)$ felt it would be necessary. Keen to keep the telephone as backup were also respondents from the United States (93\% responded 'yes', $\mathrm{n}=40$ ) and from the United Kingdom ( $89 \%$ responded 'yes', $\mathrm{n}=16$ ), but most respondents from Denmark (67\% responded 'no', $n=4)$ and Finland $(53 \%$ responded 'no', $n=9)$ did not think it would be necessary to have the telephone as back-up. Statistical tests showed no dependency between the respondent's desire to keep the telephone as back-up and their age, gender, role or most frequently used IRT system.

The respondents were asked to give their perceived reasons for preferring either the telephone or the web interface. An overview of the respondents' reported reasons is presented in Table 2 . 
The most commonly reported reasons for preferring the web interface over the telephone were practicality, easiness and time factors (Table 2). Approximately one fourth of the recipients stated that one reason for preferring the web interface over the telephone is that they have only used the web interface before. However, based on the background questions, $53 \%$ of the respondents had not used the telephone interface. This apparent discrepancy in the responses might be caused by not all respondents feeling that not trying the telephone was an actual reason for them to prefer the web interface. Of the respondents, $12 \%$ reported they prefer the web interface because some transactions are not available in the telephone interface. Obviously, this type of transaction arrangement in an IRT system would strongly direct the users towards the interface that contains the most transactions. This is a topic that might need to be addressed with the IRT service providers, as in principle a user should be able to perform all the necessary transactions via all the interfaces that are available to them within one system - apart from inherent limitations of telephone (e.g. entering free text) - or at least it should be clear to the user which transactions are available in which interface. Otherwise users may become confused with inconsistent transaction availabilities between the different interfaces, as essentially users will see the interfaces only as alternative means of accessing one single system.

The most common reason for respondents preferring the telephone interface over the web was easiness (Table 2). Some respondents also felt that the telephone interface was more easily accessible, more practical and more secure to use than the web. One respondent stated they wished to use the telephone interface because it was available in 
their local language and not only in English as the web interface. Technical issues came up, as one respondent mentioned they have had issues with the Internet registration and therefore preferred the telephone. Categorization and grouping of the open-ended responses yielded ten major themes which are presented in Table 3.

\section{Usability}

The most common theme arising from inductive analysis of the open-ended responses was usability.

The respondents felt that a visual, intuitive interface was an important factor making them prefer the web interface over the telephone. A visual interface allows an overview of the transaction being performed, as opposed to the telephone requiring the user to listen to menus and prompts. The working environment at the clinical sites was described as noisy and distracting by several respondents. The respondents felt they easily lose track if they are using the telephone, having to start over from the beginning if the call is interrupted, while the web interface allows the user to stop for a while and resume the transaction where they left off.

Many respondents felt being able to use IRT on a computer as opposed to the telephone fits better into the daily workflow at the clinical site. As defined in the ISO standard on usability, usability is a contextual concept, and in the context of clinical work, many respondents reported preferring working on the computer. ${ }^{7}$ Overall, it was considered important by the respondents that the interface of their choice should be easy and 
practical to use. These results are in line with earlier research by Viitanen et al. highlighting the usability of the systems in use in the clinical setting. ${ }^{8}$ The expectation that users might find using the telephone interface annoying or frustrating, as suggested by Settle et al. and Dyck et al. in their studies of Interactive Voice Response Systems in the general population, was also confirmed by these findings. ${ }^{9,10}$

\section{Time management}

The web interface was considered faster to use than telephone (Table 3). The perceived fastness is also probably connected with the possibility to easily combine web use with other computer work in the daily workflow. It was also highlighted in the responses that the focus at the sites is to secure as much time as possible for clinical patient work, therefore making the time management aspect of the IRT interfaces highly important.

\section{Consistency across systems}

Integrating the various technological systems being used in clinical trials brings along several benefits. ${ }^{3,11}$ The survey respondents reported suffering from the fragmented environment of multiple different systems being used in studies. The respondents feel that too many different systems are currently in use, with every system using a different logic, requiring double data entry, and the inconvenience of having to maintain multiple sets of user identification and passwords for different systems. 
Ideally there would be one single portal the user could access with a single account, containing IRT, electronic data capture (EDC), and laboratory services used within one or preferably even multiple different studies. This would be desirable from both the clinical end user and sponsor perspective, as multiple different systems require more resources to set up and maintain and more user training.

\section{Technical problems}

Technical problems with the IRT interface were felt to be more common with web than with telephone. Interestingly, this seemed to be the single area seen as a strength for telephone. The web interface is more susceptible to Internet failures, web browser issues, firewall problems in hospital networks etc., than the telephone interface which runs on telephone technology and is perceived as more reliable.

Training and user support

Some respondents wished to have more training, while others felt fewer or shorter trainings were needed. This apparent discrepancy highlights that training for IRT users needs to be well planned and implemented to cater for all kinds and levels of users. Respondents wished to have clear instructions, guidelines and forms related to the IRT systems. 
Most comments on user support were negative. The respondents wish to have immediate help if they encounter problems while a patient is waiting at the clinic, and some respondents feel they have not received adequate support in those situations.

Some of the responses reflected a need to clarify the distribution of responsibilities between the site or investigator and sponsor. The basics of the distribution of responsibilities are laid out in the International Council for Harmonisation of Technical Requirements for Pharmaceuticals for Human Use (ICH) Guideline for Good Clinical Practice (GCP), but the practical implementation may at times remain unclear in terms of IRT. ${ }^{6}$ One respondent wished as much as possible of the IRT-related work to be delegated to the sponsor, and another commented that using IRT requires more personnel resources. In other responses, an opinion was brought up that too many studies are using IRT systems in general, and that system designs do not take into account the expertise that the clinical site staff have.

These responses underline the need to clarify the core mission of conducting clinical trials at sites and the division of responsibilities between the site and sponsor. Due to Good Clinical Practice (GCP) requirements to ensure the integrity of study data, certain tasks are a site responsibility and cannot be delegated to the sponsor. Study data also needs to be independent from other patient data at the site, and therefore relying solely on hospital systems is not possible. Using a centralized IRT system is usually a prerequisite for any realistic efficient implementation of randomization and trial supply management, and it would not be foreseeable to reduce the use of IRT in clinical trials, on the contrary, it will certainly become more common in future. 
Overall the results regarding training and user support can be interpreted to reflect a need of better user support by the study sponsor and the IRT service provider. Golm et al. noticed while giving training courses to clinical study staff that site personnel often have very little knowledge about the mechanics of randomization or the wider picture how randomization, blinding, treatment allocation and IRT intertwine. ${ }^{12}$ In addition to practical end user training, these aspects should be covered to help the clinical end-users understand how IRT fits into the overall conduct of a particular study.

\section{Data errors}

Data integrity is of critical importance in clinical trials. Therefore, also the measures and processes in place to ensure the accuracy of the data are of interest to the individuals involved in generating the data. For instance, in electronic data capture (EDC) systems there are validation processes built into data entry reducing the number of errors in the entered data, and consequently also reducing the number of queries being raised after the data is submitted. ${ }^{13}$ As highlighted by Nielsen, errors are part of usability in the sense that the system should have a low error rate, so that users make few errors during the use of the system, and so that if they do make errors they can easily recover from them. ${ }^{14}$

When using the telephone interface, correcting a mistake is not always easy, as sometimes the call flow requires that the user start over from the beginning of the call. With the web interface the user has a visual overview of the entered data, reducing the 
possibility of mistakes. If the user realizes they have made a mistake in an earlier step, most web interfaces allow the user to back-track and correct the mistake before finishing the transaction.

When the transaction is already finished, and an error is spotted e.g. at data management, correcting the erroneous data can be a laborious effort, involving data clarification forms. The responses did not show a difference between the telephone and web interfaces in this respect. Therefore, it is important to focus on improving the usability of the interfaces so that the users make as few errors as possible. From this perspective, the web interface was considered superior by the respondents. When the data is correctly entered, the number of queries is reduced, and the process becomes faster and more efficient.

Language

Language is an important factor when considering the telephone and web interfaces. In current international clinical studies, the web interface is often only in English, while the telephone interface is always translated into local languages as well.

The respondents' wishes relating to English and local languages can be divided into two categories: those who wish to use English only and those who wish to use their local language when interacting with IRT systems. When a non-native speaker wishes to use English, it was considered important that the language is simple and clear to avoid misunderstandings and mistakes. Reading a foreign language is easier than listening to 
it, especially if the environment is noisy or distracting, as the clinical environment often is. Therefore, the respondents found that it is easier to use the web interface in English as opposed to the telephone interface. However, if the user wishes to use their local language instead of English, this might direct the user towards the telephone interface, as more language choices are usually available in the telephone interface. Several respondents mentioned that they would always like to have a choice of languages, and that also the web interfaces should be available in more languages.

\section{Accessibility}

Being able to access a certain IRT interface has an impact on whether the user will use it or not. Some respondents mentioned that there are limited numbers of landline phones at the clinic, but computers are always available. Thus, the web interface was seen to fit into the daily workflow better than making telephone calls by most respondents.

\section{Documentation}

International regulations provide that all parties involved in clinical trials keep records that document adequately all aspects of the conduct of the trial. ${ }^{6}$ The possibility to print screenshots and confirmations directly from the web interface was mentioned as a positive feature, as this allows more efficient and reliable documentation processes than waiting for confirmations or making manual notes from telephone interface calls. Extensive reporting options enabled by many web interfaces are also liked by the respondents. On the telephone interface the user can only perform transactions, but the 
web interface extends the functionalities to reporting and drug accountability. Thanks to its versatile functionalities, the respondents felt the web interface supports keeping electronic records better than the telephone interface.

Telephone interface as back-up

Respondents mentioned that having the telephone interface as a back-up would be wise in case the computer or Internet connection fails to work. If the transaction cannot be performed due to technical problems, the worst-case scenario is that an emergency unblinding cannot be performed in a timely manner, or that the subject does not receive their medication on time and has to return to the site for an extra visit when the transaction can be performed. This would be unethical, as according to regulations, the study subjects' safety and wellbeing should always be the primary concern in clinical trials. ${ }^{6}$ Therefore, it is critically important that IRT transactions can be performed at any time and that the database can be accessed immediately in case of a medical emergency.

\section{CONCLUSIONS}

This study provided new information about the use of IRT interfaces in clinical investigator sites. A vast majority of IRT end users at clinical sites prefer to use the web interface over the telephone interface. The most important reason behind this preference is that the overall usability of the web interface is perceived to be superior to that of telephone. Other factors considered important by IRT end users are consistency across, streamlining and integration of the various systems in use at clinical sites. Based on the 
results, it would be beneficial to improve the usability of IRT interfaces, and to increase consistency across systems. The results also highlight that support for and training of the users, as well as clarifying the responsibilities between clinical sites and the study sponsor should be a focal point for study sponsors. System streamlining and focused user training would allow a more efficient use of end user resources, yield cost savings and ultimately result in more reliable study data. The various specific functionalities of IRT systems (e.g. drug supply management, randomization and unblinding) and related interfaces would be an interesting area for future IRT research. Further research would also be beneficial in investigating the use of mobile interfaces in IRT. At the time of this study, mobile interfaces were not widely available, but this can be expected to change soon, as the IRT service providers are introducing new interfaces. While most end users want to use the web interface, many of them also feel that there would need to be a back-up interface to the web in case of unexpected circumstances preventing its use at any given time.

\section{ACKNOWLEDGEMENTS}

The authors would like to thank Dr Katja Doerk, Dr Katrin Roth and Dr Jaana Suopanki-Lalowski for their contributions.

\section{DECLARATION OF CONFLICTING INTERESTS}

The Authors declare that there is no conflict of interest. 


\section{FUNDING}

This work was supported by the Finnish Pharmaceutical Society. 


\section{REFERENCES}

1. Byrom B. Using IVRS in clinical trial management. Appl Clin Trials 2002; 11: 3643.

2. Lamberti MJ, Kush R, Kubick W, et al. An Examination of eClinical Technology Usage and CDISC Standards Adoption. Ther Innov Regul Sci 2015; 49: 869-876.

3. Haavik S. Randomization Revolution is Brewing. Appl Clin Trials 2012; 21: 3238.

4. Landau S, Everitt BS. A Handbook of Statistical Analyses using SPSS. USA: Chapman \& Hall/CRC Press LLC, 2004.

5. World Health Organization: Spotlight on statistics: Gender and health workforce statistics: Fact sheet. Issue 2: 2008.

6. International Conference on Harmonisation of Technical Requirements for Registration of Pharmaceuticals for Human Use: ICH Harmonised Tripartite Guideline. Guideline for Good Clinical Practice E6(R1). Step 4: 1996.

7. ISO: ISO 9241-210, International Standard: Ergonomics of Human-System Interaction - Part 210: Human-Centred Design for Interactive Systems, $1^{\text {st }}$ Edition 2010-03-15, Reference number ISO 9241-210:2010(E).

8. Viitanen J, Hyppönen H, Lääveri T, et al. National questionnaire study on clinical ICT systems proofs: Physicians suffer from poor usability. Int J Med Inf 2011; 80: $708-725$.

9. Settle RB, Dillon TW, Alreck PL. Acceptance of the phone-based interface for automated call direction. Behav Inf Technol 1999; 18: 97-107. 
10. Dyck JL, Emo AK, Panepinto MP. The Effect of Automated Telephone Menu Structure on User Frustration and Performance. Proceedings of the Human Factors and Ergonomics Society Annual Meeting 2007; 51: 449-454.

11. Herzberg S, Dugas M. Single source information systems can improve data completeness in clinical studies: An example from nuclear medicine. Stud Health Technol Informatics 2011; 169: 872-876.

12. Golm G, Bradstreet T, Coffey L. A course for clinical trial personnel in clinical study designs, randomization, allocation schedules, and interactive response systems. Pharm Stat 2011; 10: 175-182.

13. Pavlovic I, Kern T, Miklavcic D. Comparison of paper-based and electronic data collection process in clinical trials: Costs simulation study. Contemp Clin Trials 2009; 30: 300-316.

14. Nielsen J. Usability Engineering. New edition. USA: Morgan Kaufmann Publishers Inc., 1994. 
Table 1. Demographics of survey respondents.

\begin{tabular}{|c|c|c|}
\hline Demographics & $\mathbf{N}^{\mathbf{a}}$ & $\%$ \\
\hline \multicolumn{3}{|l|}{ Respondent's role } \\
\hline Investigator (principal or sub-investigator) & 49 & 21 \\
\hline Study Nurse / Coordinator & 118 & 51 \\
\hline Pharmacist & 50 & 22 \\
\hline Other & 14 & 6 \\
\hline All & 231 & 100.0 \\
\hline \multicolumn{3}{|l|}{ Gender } \\
\hline Female & 159 & 80 \\
\hline Male & 40 & 20 \\
\hline All & 199 & 100.0 \\
\hline \multicolumn{3}{|l|}{ Age (years) } \\
\hline $20-29$ & 29 & 15 \\
\hline $30-39$ & 73 & 27 \\
\hline $40-49$ & 49 & 24 \\
\hline $50-59$ & 39 & 20 \\
\hline $60-69$ & 9 & 4 \\
\hline All & 199 & 100.0 \\
\hline \multicolumn{3}{|l|}{ Experience of working in clinical trials } \\
\hline$<2$ years & 32 & 16 \\
\hline $2-5$ years & 54 & 27 \\
\hline $6-10$ years & 52 & 26 \\
\hline
\end{tabular}


11-15 years

$>15$ years

27

14

All

199

100.0

Country

Austria

4

2

Belgium

16

8

Canada

63

Czech Republic

10

4

Denmark

6

3

Finland

17

8

France

11

6

Germany

27

14

Italy

6

3

Poland

14

7

Spain

17

19

10

United States

46

23

All

199

100.0

Experience of using an IRT system within the past 5 years

Yes

No

All
220

95

$11 \quad 5$

231
5

100.0 
a Total number of respondents in the sample is 231 . All demographic data was captured for 199 respondents who are considered survey completers. Occasional blank answers in the background information section of the questionnaire have been ignored. 
Table 2. Survey respondents' reported reasons for preferring the web interface over the telephone interface and vice versa.

\section{Respondents' perceived reasons for preferring the web interface} over the telephone interface

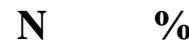

The web interface feels more practical to use than the telephone 136 65 interface

The web interface is easier to use than the telephone interface $124 \quad 59$

The web interface is faster to use than the telephone interface

The web interface is more easily accessible than the telephone interface $97 \quad 46$

$\begin{array}{lll}\text { The respondent has only used the web interface } & 55 & 26\end{array}$

The web interface feels more secure to use than the telephone interface $46 \quad 22$

Some transactions are only available in the web interface and not in the $25 \quad 12$

telephone interface

The respondent does not wish to try the telephone interface

The respondent first heard about the telephone interface when 5 2 responding to the survey

The web interface is in English while the telephone interface is in the $2 \quad 1$ local language

Other reason

126

\section{Respondents' perceived reasons for preferring the telephone interface over the web interface}


The telephone interface is more easily accessible than the web interface 2

The telephone interface feels more practical to use than the web

interface

The telephone interface feels more secure to use than the web interface $2 \quad 40$

The telephone interface is in the local language while the web interface

is in English

Other reason: "Internet interface registration takes more time than

telephone and internet tends to take a long time to process and can

time out." 
Table 3. Dimensions in open-ended responses with quotes from respondents.

Usability $(32 \%, n=74)$

Visual \& intuitive interface

"IWR is my preferred option because the visual overview of things make it a lot better."

Interface allowing frequent "Frequent interruptions: Easier to use internet interruptions \& distracting because there's no need to listen to prompts"

environment

Interface fitting the daily workflow "IWRS is more efficient to use as it is a part of everyday workflow using a computer/internet"

Ease of use \& practicality "The web-based option is easier to use and more practical."

Time management $(15 \%, n=35) \quad$ "Telephone IVRS is very time consuming"

Consistency across systems (10\%, $n=23)$

Integration of systems "Ideally: IxRS embedded into the EDC. Dosing and data management are electronic/automatically uploaded into the EDC after using the integrated IxRS."

Multiple user IDs and passwords "We use at least 27 different IWRS systems."

Technical problems $(8 \%, n=19)$ 
Internet connection

Compatibility issues

Firewalls

Reliability
"When I have issues with my computer or connection issues, that certainly personally affects my use of IxRS systems"

"I have had to use the IVR system since my current web version on my computer would not allow me to use the IWR system"

"Difficult firewalls at our hospital site" "It just seems I have had more issues with the internet than the telephone, one thing is the extra pass words and the connections are troublesome at times."

Training and user support (8\%, $n=19)$

Training "Not e-learning curses long and unuseful for pharmacists" "Training - lack of, or inadequate"

Clear instructions "Guidelines and forms that indicate which fields are required should provide more meaningful comments so the correct details are entered for example if a date is required, be specific about which date is required" 
Service provider user support

Responsibilities between site and sponsor

Data errors $(6 \%, n=15)$

Call flow

Correction of data errors

Language $(5 \%, n=12)$

Wish to use English
"I think that most important for me is contact with helpdesk. I mean that Support Team should be more helpful and should resolved the issue faster. Unfortunately sometimes I must to wait more than one day since the moment of notification." "Delegate as much as possible to monitors sponsors"

"If you accidently push the wrong keys on the phone, it takes a longer time to correct than when you make entry errors on the internet. Sometimes you have to start from the beginning and re-do everything on the phone, where on the internet you just back-track a bit and continue."

"Correcting data is often a lengthy and ver user unfriendly” english than to hear it"

"Web Interface in local language preferable" 
Simple and clear language

Choice of language

Accessibility (4\%, $n=8)$

Physical access to interface

Daily workflow

Documentation $(4 \%, n=8)$

Ability to print

Reports

Electronic records
"Clearly defined steps in basic English to avoid mistakes"

"We cannot choose our language, we are obliged to read/understand english, and not all of us are fluent." used by other people, so I cannot use the IVR system until they have finished their call. There are no eqivalent holdups with IWR."

"The internet interface is less disruptive and easily accessible."

"I can print a screenshot of the receipt confirmation immediately instead of waiting for an email or fax."

"I really like when there are reporting options that some IWRS interfaces offer" "Hopefully we'll be able to stop using paper copies of accountability logs in the future and utilise IxRS more efficiently in this capacity." 
Telephone interface as back-up to "I perceive the IWR to be easier and faster to web interface $(4 \%, n=8)$ use, but think having a back-up of IVR in case of an internet downtime is wise."

Other themes

Mobile applications "The best possible option would be the app in my phone. Do not need to have laptop or computer every time, can do something at the same time while using IWRS"

Satisfied user "I'm satisfied with IxRS interfaces now and I don't have any wishes"

Sponsor decision "Sponsor determines interface" 


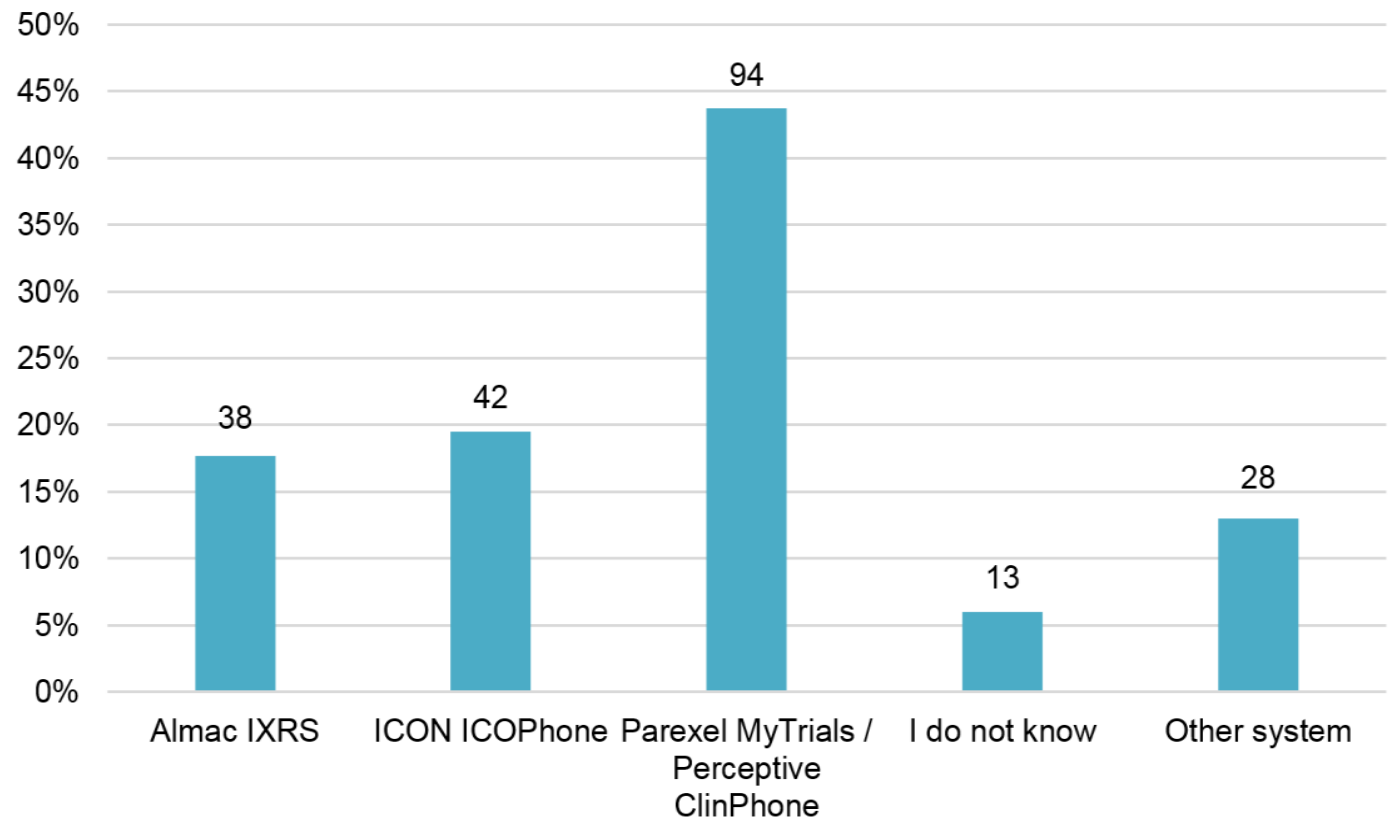

Figure 1. IRT system respondents reported using most often at the time of the survey.

Other systems mentioned included Bracket/UBC, Cenduit, Endpoint, Oracle / Phase Forward, Medidata, and Suvoda. 